supporters were Priestley and Cavendish. It continued to be the orthodox faith until the last quarter of the eighteenth century, when, after the discovery of oxygen, it was overturned by Lavoisier."

\section{Refrigeration and its Applications}

For the first of the series of Research and Development Lectures arranged under the auspices of the British Science Guild and delivered at the Royal Institution, Sir William Bragg, on May 2, took as his subject "Refrigeration". This he pointed out is of great importance to Great Britain, which imports an immense amount of meat, fish, butter and fruit, many hundreds of shiploads of which are received every year. The principles underlying refrigeration are comparatively simple, but their application on a commercial scale has involved much research such as is being carried out at Cambridge, the National Physical Laboratory, East Malling and elsewhere. Historically, the subject of heat and cold goes back to the early days of the Royal Society, and Hooke's views on fluidity are of much interest. In the eighteenth century, the theory of calorie held sway, but it was through the work of Rumford, Davy, Mayer and Joule that it was shown that heat is, in the phrase of Tyndall, a mode of motion, and to-day it can be shown that the molecules of substances are all in motion, the rapidity of which is increased by heat and decreased with cold. All the phenomena of expansion, compression and evaporation, which are utilised in refrigerating machines, are explained by this theory. Throughout the lecture, each step was illustrated by experiments in which billiard balls, bicycle pumps and liquid air played as important a part as thermo-couples and galvanometers. A singularly beautiful demonstration of the formation of vapour and clouds was given by pouring liquid air on to the surface of warm water lying in a large shallow pan. Liquid air was used also to show the alteration in the properties of substances when really cold, rubber becoming brittle and a bell of lead giving a metallic note when cooled in it. Sir William referred to the refrigeration exhibition now being held at the Science Museum, and on behalf of the director of the Museum invited all those in the audience to visit it.

\section{Electrical Phenomena at Very Low Temperatures}

Prof. J. C. McLennan gave the twenty-fifth Kelvin Lecture before the Institution of Electrical Engineers on April 26, taking as his subject "Electrical Phenomena at Very Low Temperatures". In 1823 Faraday succeeded in liquefying chlorine and afterwards succeeded in liquefying many other gases, but he failed to liquefy oxygen, nitrogen and hydrogen as he was unable to obtain the requisite low temperature. At the end of the War, a large stock of helium was available in Toronto, and this gas was successfully liquefied in 1923, a century after Faraday's experiment with chlorine. By evaporating liquid helium and thus reaching an absolute tem. perature of $0.7^{\circ} \mathrm{K}$., Keesom of Leyden successfully solidified this element in February 1932. The liquid was subjected to a pressure of 175 atmospheres and surrounded by rapidly evaporating liquid helium. The reason why liquid oxygen, hydrogen and helium are very good insulators is probably because the electrons are closely bound to the nuclei. In 1911, Kamerlingh Onnes found that the resistance of mercury vanishes suddenly at $4 \cdot 2^{\circ} \mathrm{K}$. and that some other metals behave similarly at definite low temperatures. Most metals show no trace of this supraconductivity even when great pains are taken to ensure their purity. Certain alloys have been found to become supraconductive. This supraconductivity can be destroyed by placing them in a magnetic field. The lower the temperature the greater the magnetising force necessary to destroy the supraconductivity. By suddenly destroying the magnetic field surrounding a ring of supraconductive metal, a current can be set up in it if its temperature be below the transition point. This current is quite independent of the nature of the metal and depends only on the intensity of the original induction. It looks as if the results of low temperature research would throw light on the nature of magnetism.

\section{The Restrictive Law of Population}

In his Huxley Memorial Lecture under this title, delivered on May 4, Prof. Johan Hjort, of the University of Oslo, dealt with a subject which exercised a decisive influence upon the thought of Huxley : the question of over-population (London : Macmillan and Co., Ltd. 1s. net). Prof. Hjort assumes that human society can be studied as a historical group of diverse individuals living in a restricted complex environment, and shows that biology has disclosed the many and various factors which influence the vital processes of the individuals comprising a population and determine the quantity and quality of the population as a whole. He defines an optimum population as the minimum number of individuals who can utilise to the full the vital possibilities made available by one or other of these factors. Incidentally, he surveys the fishing and whaling industries as examples, and illustrates his point that the conditions in both depend upon the power of regeneration shown by the stock. In the case of the whale, technical developments have produced a grave disharmony between the reproductive rate and the death rate, and the problem before the industry is that of defining the optimum catch. Restriction of the numbers killed is urgently demanded, but this requires both State intervention and international agreement.

According to Prof. Hjort, the ideal of all social endeavour is the maintenance of the population in a state of permanent equilibrium under conditions of life which are optimal. For the achievement of this ideal, society must undertake vast and prolonged biological experimentation. Through biology there has come an emancipation from mental chaos and from the belief that human life is governed by irrational chance. Biology has shown that overpopulation, which inevitably arises in certain given natural conditions, is not due to a superficial turmoil of moods and sentiment, but to the operation of natural laws. To-day society has both the knowledge 